\title{
Influence of Curing Process Parameters on Quality of Electrically Conductive Joints
}

\author{
Seba Barto, Pavel Mach \\ Czech Technical University in Prague, Faculty of Electrical Engineering, Department of Electrotechnology \\ Prague, Czech Republic \\ mach@fel.cvut.cz
}

\begin{abstract}
Curing parameters of electrically conductive adhesives are the temperature and the time. These parameters are recommended by a manufacturer of adhesive. The goal of the work is to find which of these parameters is more important for achievement of the low electrical resistance and low nonlinearity of a current vs. voltage characteristic of an adhesive joint. A method of factorial experiments of the type $2^{2}$ and Taguchi orthogonal array of the type L4 were used for this study. It was found that the joint resistance is mostly influenced by the curing time, less by the curing temperature and the lowest influence has the interaction of the time/temperature. Nonlinearity is also influenced mostly by the curing time and less by the curing temperature and by the interaction time/temperature.
\end{abstract}

\section{INTRODUCTION}

The electrical resistance and nonlinearity of a current vs. voltage characteristic of conductive joints formed of electrically conductive adhesives with isotropic electrical conductivity are influenced by concentration and properties of filler particles, by parameters of a coating process, and by parameters of a curing process, too. Basic parameters of a curing process are recommended by a manufacturer. Information about significance of individual parameters is missing usually.

Curing process causes chemical changes of binder of adhesive. Epoxy resin is used as binder usually. Curing process causes that binder is changed in solid. Conductive particles of filler are randomly distributed in epoxy matrix. Contacts among these particles create conductive net in adhesive.

The resistance of conductive net in adhesive depends on following factors:

- On chemical layers, created during adhesive curing, which cover filler particles, decrease contact conductivity and increase its noise and nonlinearity of the current vs. voltage characteristic.
- On the shape of filler particles. It has been found that conductive net in adhesive filled with flakes has lower resistance than conductive net in adhesive filled with balls, if concentration both types of these particles is the same (by weight).

- On the material of filler, especially on its electrical resistance. The temperature dependence of filler resistance influence the temperature dependence of adhesive bonds significantly.

- On the force overpressing particles to each other. The higher is this force; the better is quality of contacts between them. It has been found that curing of epoxy resin causes decrease of its volume by $7 \%$ [1]. This shrinkage contributes to improvement of contacts quality.

The goal of the work has been to examine the influence of a curing process of electrically conductive adhesive on basic electrical parameters of adhesive joints. These parameters are the joint resistance, noise and nonlinearity of the current vs. voltage characteristic. Therefore a mathematical model of the curing process has been calculated. Analysis of the model will make optimization of curing process parameters possible. 
Curing process is described by two parameters: by the temperature and by the time of curing. In accordance with the theory, for calculation of a mathematical model the parameters must also be completed with interaction of these parameters that means with interaction temperature/time. The reason is that such the interaction can cause synergic effect on cured adhesive.

\section{EXPERIMENTAL}

Ag filled bisphenol A epoxy resin with isotropic electrical conductivity was used for fabrication of test samples. Adhesive was applied by dispensing.

SMT jumpers were mounted using adhesive on a test board. The layout of a test board is shown in Fig. 1. Ten test boards with seven mounted jumpers were fabricated.

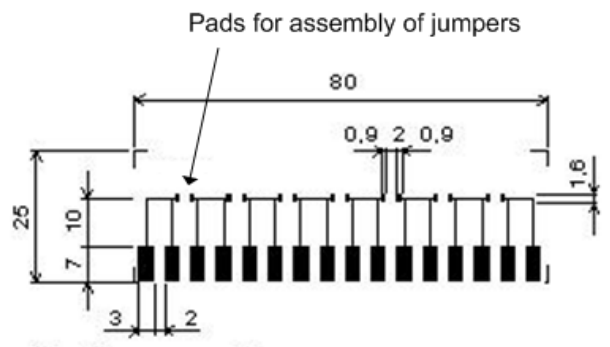

Fig. 1. Layout of test board for adhesive assembly

A four-terminal method was used for the measurement of the adhesive contacts resistances and nonlinearity of the current vs. voltage characteristic of these contacts. The LCR meter HP 4284 was used for the resistance measurements, special equipment developed at the Department of Electrotechnology, was used for nonlinearity measurements.

\section{THEORY}

With respect to investigation of two output parameters of the process - the joint resistance and joint nonlinearity, two mathematical models have been calculated. Noise has not been investigated, because the noise level is very low and its measurement needs a specially screened room and equipment.

The mathematical models have been found by factorial experiments [2].
Schematic diagrams of factorial experiments for both the models can be seen in the paper "Tolerance Design of Curing Process of Electrically Conductive Adhesives" in this proceeding. An example of the diagram for calculation of the model for the joint resistance is shown in Tab. 1.

Tab. 1. Schematic diagram of factorial experiments for calculation of the model for the joint resistance

\begin{tabular}{|c|c|c|c|c|}
\hline \multirow{4}{*}{ 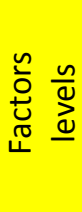 } & \multicolumn{2}{|c|}{$A 1=120$} & \multicolumn{2}{|c|}{$A 2=170$} \\
\hline & $B 1=20$ & $B 2=80$ & $B 1=20$ & $B 2=80$ \\
\hline & $A 1 B 1$ & $A 1 B 2$ & $A 2 B 1$ & $A 2 B 2$ \\
\hline & (1) & $b$ & $a$ & $a b$ \\
\hline \multirow{5}{*}{ 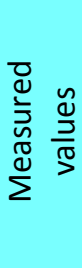 } & $y_{1,1}=32.5$ & $y_{2,1}=20.3$ & $y_{3,1}=12.3$ & $y_{4,1}=13 \cdot 2$ \\
\hline & $y_{1,2}=28.4$ & $y_{2,2}=18.1$ & $y_{3,2}=14.7$ & $y_{4,2}=16.4$ \\
\hline & $y_{1,3}=26.2$ & $y_{2,3}=21.4$ & $y_{3,3}=15.1$ & $y_{4,3}=12 \cdot 9$ \\
\hline & $y_{1,4}=37.9$ & $y_{2,4}=19.7$ & $y_{3,4}=11.4$ & $y_{4,4}=24.7$ \\
\hline & $y_{1,5}=34.8$ & $y_{2,5}=16.5$ & $y_{3,5}=13.5$ & $y_{4,5}=13.5$ \\
\hline$\Sigma$ & R1 & R2 & R3 & R4 \\
\hline
\end{tabular}

Where $A \ldots$ temperature $\left({ }^{\circ} \mathrm{C}\right), B \ldots$ time $(\min )$, $a, b \ldots$ symbolic variables. $R_{i}$ is calculated using a formula:

$$
R_{i}=\sum_{j=1}^{r} y_{i, j}
$$

Where $y_{i, j} \ldots$ measured value in $\mathrm{i}$-th column and $\mathrm{j}$-th raw, $r \ldots$ number of rows (it means that every experiment has been $\mathrm{r}$-time repeated).

Symbolic variables (1), $a, b$ are used for theoretical description in equations for derivation of the mathematical model. These variables are substituted with the column sums $R_{i}, i=1, \ldots, 4$ (see Tab. 1) for final calculation.

First estimation of influence of technological factors levels on the level of the output parameter must be calculated. These influences are calculated as differences between the values of the output parameter for upper and lower level of an input parameter.

It is possible to write for the factors $A$ and $B$ in symbolic expression:

$$
\begin{aligned}
& Z_{A}=(a+a b)-((1)+b) \\
& Z_{B}=(b+a b)-((1)+a)
\end{aligned}
$$


Following equation has been found for interaction of factors $A$ and $B$ :

$$
Z_{A B}=((1)+a b)-(a+b)
$$

Sums of squares of deviations were calculated using formulas:

$$
S_{A}=\frac{Z_{A}{ }^{2}}{d . r}, S_{B}=\frac{Z_{B}{ }^{2}}{d . r} \quad, S_{A B}=\frac{Z_{A B}{ }^{2}}{d . r}
$$

Where $d$... number of columns, $d=2^{n}, n \ldots$ number of technological factors

Significance of the factors $A$ and $B$ and interaction $A B$ has been tested using an F-test. Test characteristics for the factors and interaction have been calculated according to the equations:

$$
F_{A}=\frac{S_{A}}{S_{R} / v}, \quad F_{B}=\frac{S_{B}}{S_{R} / v}, \quad F_{A B}=\frac{S_{A B}}{S_{R} / v}
$$

Where $v \ldots$ degrees of freedom.

Calculated values of $F_{k}$, where $\mathrm{k} \equiv A, B$, or $A B$, have been compared with the critical value of $\mathrm{F}$ distribution $F_{\alpha}(1, v)$, where $\alpha \ldots$ level of significance. It has been found that all three values of $F_{k}$ are higher than the value of $F_{\alpha}$. Therefore both the parameters and interaction $A B$ must be involved into the model.

Then following transformation has been carried out for the factor $A$ :

$$
x_{1}=\frac{2}{A_{2}-A_{1}} \cdot\left(A-\frac{A_{1}+A_{2}}{2}\right)
$$

Analogical transformation have also been carried out for the factor $B$ (the transformed variable has been marked $x_{2}$ ).

Coefficients $b$ of the linear model

$$
y=b_{0}+b_{1} x_{1}+b_{2} x_{2}+b_{12} x_{1} x_{2}
$$

have been calculated using the method of least squares. Following formulas for these coefficients have been derived as the result of the solution of normal equations:

$$
b_{0}=\frac{1}{d} \sum_{i=1}^{d} \overline{y_{i}}
$$

Where $\overline{y_{i}} \quad \ldots$ arithmetic average of values measured in i-th column, $\overline{y_{i}}=R_{i} / r$

$$
b_{1}=\frac{Z_{A}}{d \cdot r}, \quad b_{2}=\frac{Z_{B}}{d \cdot r}, \quad b_{12}=\frac{Z_{A B}}{d \cdot r}
$$

Quality of the model has been tested using an F-test. The test characteristic was calculated according to the formula:

$$
F=\frac{\frac{S_{S}}{d \cdot r-n-1}}{\frac{S_{r}}{v}}
$$

Where $S_{s} \ldots$ sum of squares of deviations between measured values and the mathematical model:

$S_{S}=\sum_{i=1}^{d} \sum_{j=1}^{r}\left(y_{i, j}-b_{0}-b_{1} x_{1, i}-b_{2} x_{2, i}-\ldots-b_{n} x_{n, i}\right)^{2}$

and $S_{r} \ldots$ residuum sum of squares:

$$
S_{r}=\sum_{i=1}^{d} \sum_{j=1}^{r}\left(y_{i, j}-\bar{y}_{i}\right)^{2}
$$

Degrees of freedom $v$ have been calculated using the equation:

$$
v=d \cdot(r-1)
$$

Calculated value $F$ has been compared with the critical value of F-distribution $F_{\alpha}(d . r-n-1, v)$. It has been found that the calculated values of $F$ have been lower than values $F_{\alpha}$ for both the models, for the model for the joint resistance calculation as well as for the model for joint nonlinearity calculation. It means that the models have sufficient accuracy.

Following models have been found:

The model for the joint resistance:

$$
y=20,175-5,405 x_{1}-2,505 x_{2}+3,875 x_{1} x_{2}
$$

The model for joint nonlinearity:

$$
y=6,82-1,015 x_{1}-2,215 x_{2}-0,825 x_{1} x_{2}
$$

Then the models have been transferred in models for technological factors $A$ (the curing temperature in ${ }^{\circ} \mathrm{C}$ ) and $B$ (the curing time in min.) using reverse transformation..

1. Model for the joint resistance:

$R=68,794-0,4742 A-0,8835 B+0,0052 A B$

2. Model for joint nonlinearity:

$U_{\text {nelin }}=1,46+0,0144 A+0,0967 B-0,0011 A B$ 
Both the models are valid for following specification limits:

$$
\begin{aligned}
& \text { temperature }\left({ }^{\circ} \mathrm{C}\right) \equiv<120,170> \\
& \text { time }(\min ) \quad \equiv<20,80>.
\end{aligned}
$$

The data were also processed using a Taguchi table L4 [3]. The structure of this table is the same like the structure of the table for factorial experiments of the type $2^{2}$. Significance of the curing process parameters for the value of the joint resistance and nonlinearity has been examined using this table [4]. Results are expressed graphically in Fig. 2 and Fig. 3.

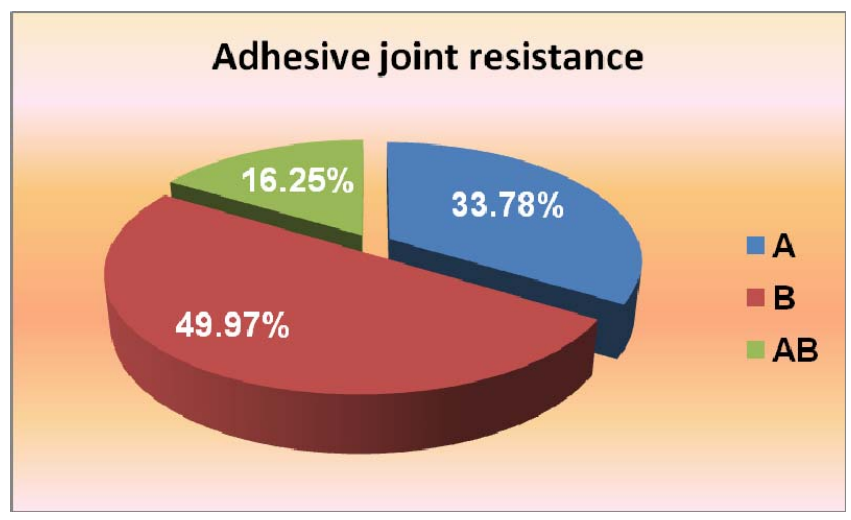

Fig. 2. Influence of curing parameter $A$ (the temperature in ${ }^{\circ} \mathrm{C}$ ), $B$ (the time in min.) and their interaction $A B$ on the adhesive joint resistance

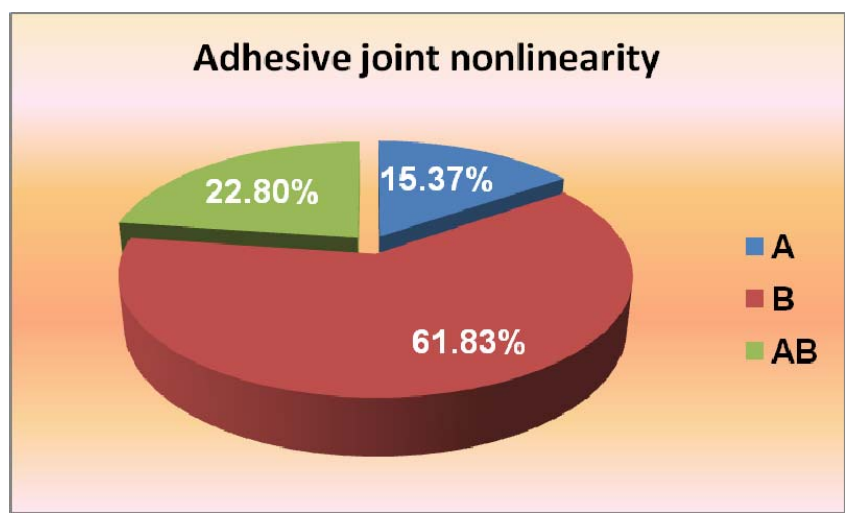

Fig. 3. Influence of curing parameter $A$ (the temperature in

${ }^{\circ} \mathrm{C}$ ), $B$ (the time in min.) and their interaction $A B$ on nonlinearity of the current vs. voltage characteristic of adhesive joint

Surprising is the magnitude of influence of interaction $A B$ on the result of the curing process. This is similar to ageing of epoxy resin in the temperature, in humidity and in combination temperature/humidity.

\section{Discussion of Results}

The models make calculation of electrical parameters of the joints in dependence on the curing parameters possible. Statistical testing has shown that they have good conformity with measured results. The models have shown that the curing temperature has lower influence on the electrical parameters of the joint than the curing time. The reason, why the curing temperature has lower influence than the curing time, depends on the principle of mechanisms caused by the curing process. These mechanisms can differ in dependence on the type of resin and in dependence on the interval of the curing temperature. Therefore the models are valid for specification limits, for that the experiments have been carried out, and for resin under the test only. The specification limits have been defined by a manufacturer of adhesive.

\section{Conclusions}

Mathematical models for basic electrical parameters of adhesive joints in dependence on the curing process parameters have been calculated using DOE. Significance of curing factors - the temperature and the time, has been examined using Taguchi approach. The models are in good accordance with the measured results. They are usable for technological conditions, for which they have been calculated. The Taguchi analysis has shown that the curing time is more significant than the curing temperature for values of electrical parameters of the joints.

\section{ACKNOWLEDGMENTS}

The work has been carried out as a part of a project "Diagnostics of Materials", number MSM6840770021

\section{REFERENCES}

[1] Su, B.: „Electrical, Thermomechanical and Reliability Modeling of Electrically Conductive Adhesives", Disertation. Georgia Institute of Technology. 2006

[2] MONTGOMERY, D. C.: "Introduction to Statistical Quality Control", J. Wiley and Sons, 2001

[3] TAGUCHI, G.:"Introduction to Quality Engineering", American Supplier Institute, 1986

[4] TAGUCH, G., CHOWDHURY, S., WU, Y.: „Taguchi's Quality Engineering Handbook", John Wiley and Sons, 2005 